\title{
DRIVER SIMULATION-BASED TRAINING OF HEAVY VEHICLE OPERATORS: TARGETED TASK ANALYSIS AND CONSIDERATIONS FOR TRAINING DESIGN
}

\author{
Eve Mitsopoulos-Rubens ${ }^{1}$, Michael G. Lenné ${ }^{1} \&$ Paul M. Salmon ${ }^{2}$ \\ ${ }^{1}$ Monash University Accident Research Centre, Monash University, Melbourne, Victoria, \\ Australia \\ ${ }^{2}$ University of the Sunshine Coast, Queensland, Australia \\ Email: eve.mitsopoulos@monash.edu
}

\begin{abstract}
Summary: The use of simulation for training operators of heavy vehicles is gaining momentum. However, there still exists a gap in knowledge about the appropriate skills to target, and in particular, with regards to skill areas of a nontechnical nature. By taking a first-principles approach, we first sought to conduct a targeted analysis of the heavy vehicle operator task and, in turn, to assess which of the goal-based tasks identified through the task analysis would be most appropriate for simulation-based training. In general, simulation provides a safe and efficient option for training critical skills that could otherwise be trained on road (e.g., gear shifting). Simulation also provides the opportunity to train critical skills in a structured and formal way that could otherwise not be achieved in a real heavy vehicle, except on an opportunistic or incidental basis (e.g., hazard perception). Nonetheless, the challenge for training system design still remains: what constitutes the appropriate balance between simulator-based and real truckbased practical training, and for which curriculum components and skill sets.
\end{abstract}

\section{INTRODUCTION}

Heavy vehicles play a central role in the transport of freight and provision of services. Safe and efficient operation of a heavy vehicle requires mastery of specialised knowledge and skills (Blanco, Hickman, Hanowski \& Morgan, 2011). Training provides one mechanism through which certain critical competencies can be imparted and developed. Constraints on the time available for training, the ongoing operation and maintenance costs associated with the use of real vehicles for training, and the safety risks to the trainer, trainees and other road users associated with training in the real world environment, highlight the need for training providers and organisations with heavy vehicle fleets to explore options for the safe and cost-effective training of heavy vehicle operators. Simulation constitutes one such option.

There is now much evidence in support of simulators for the training of operators in several transportation modes. While a role for simulation in civil and military aviation is wellestablished, critically, there is emerging evidence pointing to the efficacy of simulation for training heavy vehicle operators (e.g., Morgan, Tidwell, Medina \& Blackwell, 2011) and, in particular, in the principles of eco-driving (e.g., Reed, Cynk \& Parkes, 2010).

A thorough appreciation of the task to be trained can provide the basis for developing an understanding of the training needs of a given group of operators. These training needs, in turn, can be considered in terms of what media, methods and techniques would be best suited to addressing them. With this in mind, we conducted a targeted analysis of the heavy vehicle operator task. Consideration was given to the sorts and range of technical (e.g., vehicle control 
and manouvreing) and non-technical (e.g., hazard perception) skill areas in which experienced operators of heavy vehicles are expected to be sufficiently proficient in order to operate their vehicle safely and efficiently. In turn, an assessment was made of which tasks identified through the analysis would be most suitable for training using simulation. In this paper, we present the key outcomes of the task analysis, and offer some recommendations for which tasks would be the most appropriate to target in simulation-based heavy vehicle operator training. For current purposes, the type of simulator in mind was a closed-loop, fully interactive system, with at least mid-range levels of physical fidelity, and with both part-task and full-task capabilities.

The work presented here forms part of a broader research project in to simulation-based training for heavy vehicle operators that was undertaken for an Australian-based training provider. Other project activities included a targeted literature review, and a gap analysis between the recommendations deriving from the task analysis and the simulation-based training currently available through the provider. It is appreciated that work with some overlap in overall goals has been conducted in jurisdictions elsewhere. However, the extent to which this previous work can be extrapolated to the Australian context is not yet clear. This is an important gap in knowledge as jurisdictions may differ in their requirements for heavy vehicle operator training, stemming, at least in part, from differences in licensing regimes, the motivations and needs of organisations with heavy vehicle fleets, and fundamentally, the resources available. Thus, the approach adopted in the current research was considered appropriate, with the added advantage of helping both to validate and extend the important work which had previously been done in this area.

\section{TASK ANALYSIS OF HEAVY VEHICLE OPERATION}

Hierarchical Task Analysis (HTA) was the task analysis technique adopted in the current research. HTA is, in essence, a goal-based representation of tasks performed by a system. A given task is decomposed into its component goals and sub-goals, thus reflecting the hierarchical relationship between them. For a given hierarchy, a "plan” is specified, with that plan defining the conditions under which the sub-goals of a super-ordinate goal are triggered (Stanton, 2006). The degree and nature of task decomposition is determined by the HTA's overall purpose. Thus, a given HTA may not represent the definitive and ultimate analysis of a given system.

To develop the HTA in the current research, Stanton's (2006) nine heuristics were followed. The current HTA was conducted to facilitate identification of the training needs of heavy vehicle operation that could, in turn, be reviewed for their ability to be trained through simulators of given characteristics. The focus of the HTA was on the operations carried out by the individual vehicle operator - that is, the driver in a crew of one. It was imperative that the current HTA of heavy vehicle operation cover both technical and non-technical skill areas. It was also important that the HTA explicitly cover the principles of eco-driving. The main data sources were of three types: (i) observations of, and discussions with, experienced operators of heavy vehicles; (ii) training course documentation, and the relevant licensing authority's truck driver's handbook; and (iii) relevant literature, including any previous HTAs of vehicle operation. The HTA was developed with trucks (rigid, heavy and multi-combination) with manual (synchromesh and nonsynchromesh) transmission in mind; however, the analysis could be readily applied to heavy vehicles with automatic transmission. Given the magnitude of the final HTA, it is not possible to present it in full here. An extract of the HTA, excluding plans, is given in Table 1. This extract is 
intended to highlight the breadth of the HTA rather than its depth and so, the decomposition of sub-goals is shown to either two or three levels deep only.

Table 1. High-level extract of HTA of heavy vehicle operation

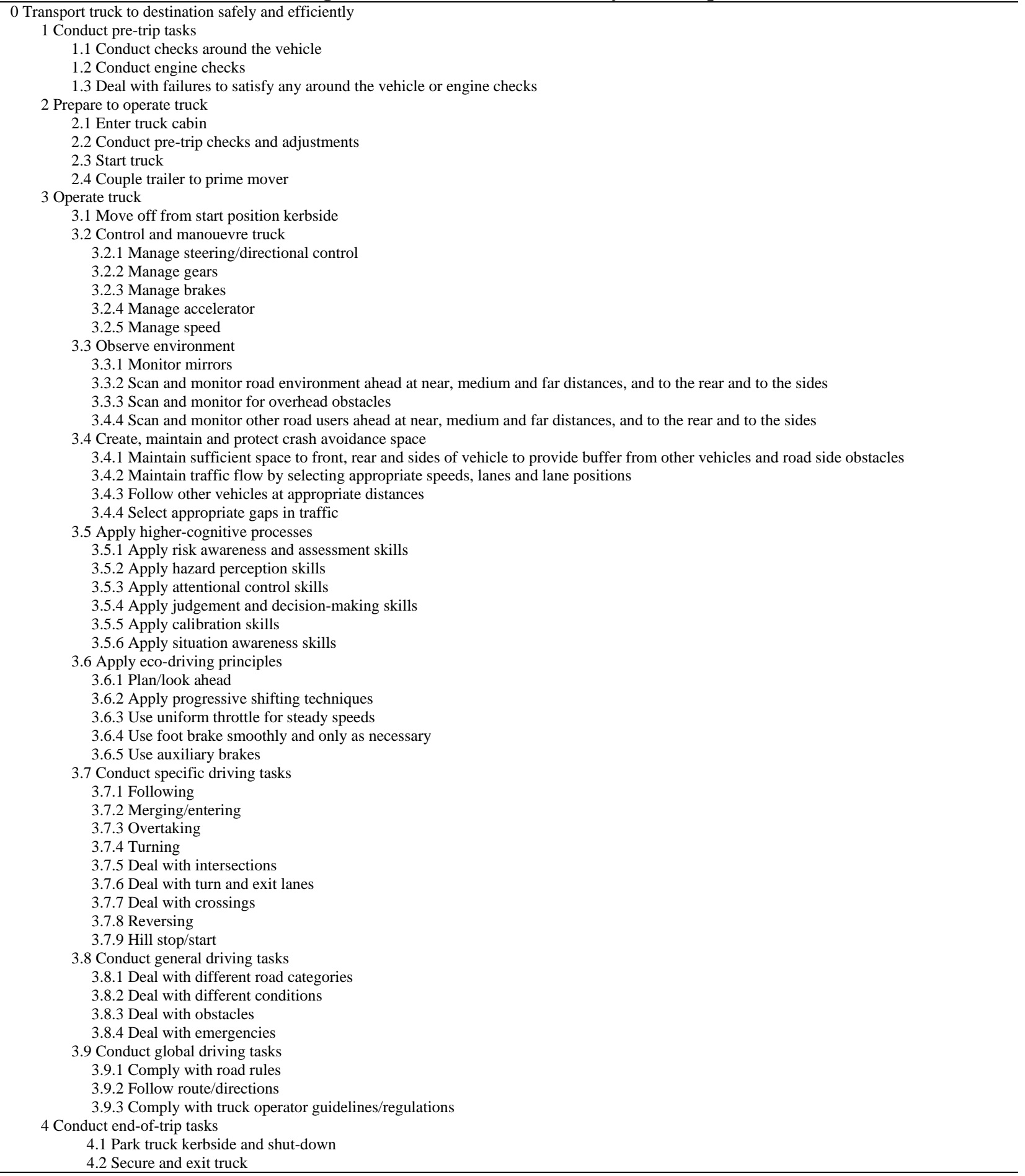

Table 1 shows that heavy vehicle operation for the single-member crew has several components. While driving the truck is a significant component, the HTA also covers critical pre-trip checks 
and adjustments, and other preparatory tasks, such as, where relevant, coupling the trailer to the prime mover. Technical and non-technical skill areas are embedded within the HTA, with many of the sub-goals drawing on both - for example, sub-goal 3.4 "create, maintain and protect crash avoidance space” and sub-goal 3.7 “conduct specific driving tasks”. Specific technical and nontechnical skill areas are also highlighted and decomposed as appropriate, with a certain level of proficiency in these areas implied in the execution of other sub-goals. Noteworthy specific technical skill areas are covered mostly in sub-goal 3.2 “control and manouevre truck”, while specific, quintessential, non-technical skills areas are considered, in part, under sub-goal 3.5 “apply higher-cognitive processes”. Sub-goal 3.5 "apply higher-cognitive processes” was decomposed into six sub-goals. While not necessarily mutually exclusive, these six skill areas are referred to explicitly here. The listing here of non-technical skills additional to hazard perception (e.g., attentional control) is particularly noteworthy, given that these skills are often overlooked in training programs, despite their importance to safe driving. Of further relevance here is that these skills have been identified as being potentially amenable to simulation-based training methods (Triggs, 1994). A dedicated sub-goal was created to deal explicitly with ecodriving. Based largely on the available documentation and relevant literature, sub-goal 3.6 “apply eco-driving principles” was decomposed into five sub-goals.

It is important to note that goal-based tasks pertaining to compliance with organisational or regulatory requirements have not be covered at all in the HTA or covered only partially or tangentially. This was deliberate given that the focus of the HTA was on identifying or emphasizing those tasks that would be most suitable to simulation-based training using a simulator of given characteristics. Were the HTA undertaken for another overarching purpose for example, to delineate in turn the full training needs of heavy vehicle operators within a given organisational context - then a slightly different goal/sub-goal hierarchy may have emerged that included greater decomposition and delineation of organisation and government-driven tasks and requirements for heavy vehicle operators. This issue reinforces the point made earlier that a given HTA may not provide the definitive representation of a given system. The usefulness and validity of a HTA needs to be considered in the context for which it was developed.

\section{IDENTIFACTION OF TASKS SUITABLE FOR SIMULATION-BASED TRAINING}

Based on a review of the literature on the use of simulation for heavy vehicle operator training, and of the literature regarding the development of skilled performance in general and in driving specifically, an assessment was made on which goal-based tasks identified through the HTA would be most suitable to training using simulation as a medium. As noted earlier, the type of simulator in mind was a closed-loop, fully interactive system, with at least mid-range levels of physical fidelity, and with both part-task and full-task capabilities. Table 2 summarises the outcomes of the assessment.

Table 2 distinguishes between those goal-based tasks that would not be an appropriate target for simulation ( $\boldsymbol{x}$ ), would be an appropriate target $(\checkmark)$, and those that would be a highly appropriate target for simulation $(\checkmark \checkmark)$. Tasks that would be highly appropriate to target for simulation include technical and non-technical skill areas. There would be perhaps little disagreement that addressing the basic knowledge and skills associated with controlling and manouvreing a truck would be a useful starting point for simulator-based training at the entry-level. The approach, in 
general, would be to increase task demand and complexity with increasing proficiency in the skills being trained. It is noteworthy that, as part of the US-based "SimVal" research program, Morgan, Tidwell, Medina, Blanco, Hickman and Hanowski (2011) reported that participants who were trained in the simulator rated "basic control" as the most effective training feature of the simulator.

Giving trainees the opportunity to be exposed to, and practice, the full range of situations with which they might be presented on the road throughout their career, but may not have the opportunity to experience in the short-term as part of an on-road practical training component, constitutes a further worthy target of simulation. Use of simulation in this sense would also be appropriate as part of routine refresher training efforts. Interestingly, the findings generally

Table 2 Goal-based tasks identified as most suitable for simulation-based training

\begin{tabular}{|c|c|c|}
\hline Sub-goal from the HTA & $\begin{array}{l}\text { Target for } \\
\text { simulation? }\end{array}$ & Description \\
\hline $\begin{array}{l}\text { Conduct checks around the } \\
\text { vehicle (1.1) }\end{array}$ & $x$ & \\
\hline Conduct engine checks (1.2) & $x$ & \\
\hline Deal with failures (1.3) & $x$ & \\
\hline Enter truck cabin (1.4) & $x$ & \\
\hline $\begin{array}{l}\text { Conduct pre-trip checks and } \\
\text { adjustments (2.2) }\end{array}$ & $x$ & \\
\hline Start truck (2.3) & $\checkmark$ & $\begin{array}{l}\text { A particular focus here could be on checking instruments and gauges. Could include, } \\
\text { if possible, simulating unexpected or abnormal changes to give trainees exposure to } \\
\text { what these may look like and practice in identifying them. }\end{array}$ \\
\hline $\begin{array}{l}\text { Couple trailer to prime mover } \\
(2.4)\end{array}$ & $\checkmark$ & $\begin{array}{l}\text { Trainees could be given practice in reversing the prime mover to position it in front of } \\
\text { the trailer as required. }\end{array}$ \\
\hline $\begin{array}{l}\text { Move off from start position } \\
(3.1)\end{array}$ & $\checkmark$ & $\begin{array}{l}\text { Trainees could be given the opportunity to practice execution of the sequence of } \\
\text { steps, commencing with putting the truck into gear. }\end{array}$ \\
\hline $\begin{array}{l}\text { Control and maneouvre truck } \\
\text { (3.2) }\end{array}$ & $\checkmark \checkmark$ & $\begin{array}{l}\text { A particular focus here could be on managing steering/directional control, gears, and } \\
\text { brakes - e.g., } \\
\text { Manage steering/directional control (3.2.1): Focus initially on practicing monitoring } \\
\text { of road position, good steering techniques, and use of indicators with no traffic, first } \\
\text { with straight line driving, then curves and intersections. Introduce traffic when } \\
\text { appropriate. } \\
\text { Manage gears (3.2.2): Focus initially on identifying need to change gears and } \\
\text { changing gears smoothly without the visual display, then introduce scenario with no } \\
\text { traffic. Introduce traffic when appropriate and opportunities to shift up and down. }\end{array}$ \\
\hline Observe environment (3.3) & $\checkmark \checkmark$ & $\begin{array}{l}\text { Trainees could be given specific practice in monitoring mirrors, scanning in all } \\
\text { directions, and at near, medium and far distances. Techniques such as commentary } \\
\text { driving could be employed. }\end{array}$ \\
\hline $\begin{array}{l}\text { Create, maintain and protect } \\
\text { crash avoidance space (3.4) }\end{array}$ & $\checkmark \checkmark$ & $\begin{array}{l}\text { An aim here could be to give trainees specific practice in strategies they could use to } \\
\text { create, maintain and protect the space around the vehicle. }\end{array}$ \\
\hline $\begin{array}{l}\text { Apply higher-order processes } \\
\text { (3.5) }\end{array}$ & $\checkmark \checkmark$ & $\begin{array}{l}\text { Specific training modules could be devoted to providing practice in each of these } \\
\text { areas - e.g., hazard perception module developed for SCOTSIM (Reed et al, 2007). }\end{array}$ \\
\hline $\begin{array}{l}\text { Apply eco-driving principles } \\
\text { (3.6) }\end{array}$ & $\checkmark \checkmark$ & $\begin{array}{l}\text { A specific training module could be devoted to providing practice in application of } \\
\text { principles of eco-driving. }\end{array}$ \\
\hline $\begin{array}{l}\text { Conduct specific driving tasks } \\
\text { (3.7) }\end{array}$ & $\checkmark \checkmark$ & $\begin{array}{l}\text { An aim here could be to give trainees exposure to and the opportunity to practice } \\
\text { dealing with the full range of specific driving tasks - e.g., reversing, dealing with } \\
\text { intersections. }\end{array}$ \\
\hline $\begin{array}{l}\text { Conduct general driving tasks } \\
\text { (3.8) }\end{array}$ & $\checkmark \checkmark$ & $\begin{array}{l}\text { An aim here could be to give trainees exposure to and the opportunity to practice } \\
\text { dealing with the full range of general driving tasks. A particular focus could be in } \\
\text { exposing trainees to particular types of emergencies (e.g., tyre blowout) in order to } \\
\text { impart strategies and provide practice opportunity for dealing with such situations. }\end{array}$ \\
\hline $\begin{array}{l}\text { Conduct global driving tasks } \\
(3.9)\end{array}$ & $\checkmark$ & $\begin{array}{l}\text { May not be a specific focus of training, but need to comply with road rules, would be } \\
\text { an expectation of/implied in some other training modules. }\end{array}$ \\
\hline Park truck and shut down (4.1) & $\checkmark$ & $\begin{array}{l}\text { Trainees could be given the opportunity to practice execution of the sequence of } \\
\text { steps. }\end{array}$ \\
\hline Secure and exit truck (4.2) & $x$ & \\
\hline
\end{tabular}


As noted above, with the exception of hazard perception, higher-order processes have not received much attention in the domain of heavy vehicle operator training. While there may be some transfer of these skills from car driving to truck driving in a general sense, the inherent differences between driving a car and driving a truck (e.g., more complicated controls, different driver view-point, greater size, heavier load), would suggest a need for targeted training in these areas, and even at the entry-level. The development of modules additional to the eco-driving training module as part of the SCOTSIM research program (Reed, Parkes, Peacock, Lang \& Rehm, 2007) is worth acknowledging. While not formally evaluated, information on these modules provides some insight into how specific simulator-based training into higher-order processes can be achieved. The module on hazard perception is particularly noteworthy here. As described by Reed et al. (2007), the training scenarios are of three types: (1) entry of another object into the path of the driven vehicle; (2) unexpected actions of the vehicle in front; and (3) hazardous actions of oncoming traffic. Trainees are exposed to these scenarios across four exercise types: rural roads, urban roads, and two motorway. The goal of the training is to develop trainees’ ability to detect and identify hazards early and to adapt their driving performance accordingly.

\section{DISCUSSION}

Based on the assessment of goal-based tasks that are most suitable to simulation-based training, it appears that there are two general ways through which simulation can be used as a medium to impart heavy vehicle operator training. The first is to train, safely and efficiently, critical skills that could otherwise be trained on-road. The second aspect is to train critical skills in a targeted and structured way that could otherwise not be achieved, except perhaps on an opportunistic or incidental basis, in a real truck. While this distinction may not be a novel one, it is still an important one to emphasise. Where simulation has been used in Australia to train heavy vehicle operators at the entry-level, the prevailing tendency has been to focus on basic elements of vehicle control. To help capitalize on the benefits that simulation has to offer, simulation-based training programs, even those at the entry-level, should look to address both elements.

Simulation has many advantages to offer over training in an actual vehicle, including safety, efficiency, flexibility, repeatability and consistency, and the ready provision of performance feedback based on objective data. However, incorporating simulation into a training program is by no means a trivial undertaking. While guidance exists on how best to match simulator capabilities with training needs for heavy vehicle operators (e.g., Brock, Jacobs, Van Cott, McCauley \& Norstrom, 2001), there is still a gap in knowledge regarding how much simulation (i.e., what proportion of a broader training program is simulation-based) is both necessary and sufficient to maximise the overall effectiveness of a training program. In the absence of such knowledge, the issue is typically addressed on pragmatic grounds. This then leads to the question of where should the focus of the simulator-based training component lie given a simulator platform of given characteristics, the length of time that can reasonably be allocated to simulatorbased training, the purpose of the training, and the experience level of the target trainee audience. In essence, the question can be reduced to one of how much simulation, for what, and for whom? However, in addressing this multi-faceted question, consideration needs to be given to what constitutes the appropriate balance between simulator-based and real truck-based practical training, and for which curriculum components and skill sets. 


\section{CONCLUSION}

The research presented here offers a taxonomy of which goal-based tasks of heavy vehicle operation would be the most suitable to train through simulation-based methods. However, a critical issue is that, for best effect, the use of simulation as a training tool needs to be integrated appropriately into the total training curriculum. Further transfer of training to the real world, along with other measures of training quality (e.g., skill retention) should be formally evaluated and ideally, through the collection and analysis of objective performance data.

\section{ACKNOWLEDGMENTS}

The research activities described in this paper were undertaken as part of a larger project for Driver Education Centre of Australia, Ltd. Their support is gratefully acknowledged.

\section{REFERENCES}

Blanco, M., Hickman, J.S., Hanowski, R.J., \& Morgan, J.F. (2011). The commercial driver. In Fisher, D.L., Rizzo, M., Caird, J.K., \& Lee, J.D. (Eds.), Handbook of driving simulation for engineering, medicine, and psychology (pp. 31-1-31-12). Boca Raton, FL: CRC Press.

Brock, J.F., Jacobs, C., Van Cott, H., McCauley, M., \& Norstrom, D.M. (2001). Simulators and bus safety: Guidelines for acquiring and using transit bus operator driving simulators (Report No. TCRP Report 72). Washington, DC: Transportation Research Board.

Morgan, J.F., Tidwell, S., Medina, A., \& Blanco, M. (2011). On the training and testing of entrylevel commercial motor vehicle drivers. Accident Analysis and Prevention, 43, 14001407.

Morgan, J.F., Tidwell, S.A., Medina, M., Hickman, J.S., \& Hanowski, R.J. (2011). Commercial motor vehicle driving simulator validation study (SimVal): Phase II (Report No. FMCSA-RRR-11-014). Washington, DC: US Department of Transportation, Federal Motor Carrier Safety Administration.

Reed, N., Cynk, S., \& Parkes, A.M. (2010). From research to commercial fuel efficiency training for truck drivers using TruckSim. In Dorn, L. (Ed.), Driver behaviour and training, volume IV (pp. 257-268). Aldershot, UK: Ashgate

Reed, N., Parkes, A.M., Peacock, C., Lang, B., \& Rehm, L. (2007). SCOTSIM: an evaluation of the effectiveness of two truck simulators for professional driver training (Report No. PPR214). Transport Research Laboratory, Crowthorne, UK.

Stanton, N.A. (2006). Hierarchical task analysis: developments, applications and extensions. Applied Ergonomics, 37, 55-79.

Triggs, T.J. Human performance and driving: The role of simulation in improving young driver safety. Proceedings of the $12^{\text {th }}$ Triennial Congress of the International Ergonomics Association (Vol. 1, pp. 23-26). Toronto, Canada. 